\title{
Chaotic behavior in a class of delay difference equations
}

\author{
Zongcheng Li*, Qingli Zhao and Di Liang
}

${ }^{*}$ Correspondence:

lizongcheng_0905@yahoo.com.cn

Department of Mathematics,

Shandong Jianzhu University, Jinan,

Shandong 250101, P.R. China

\begin{abstract}
In this paper, we rigorously prove the existence of chaos in a class of delay difference equations, which can be viewed as a discrete analogue of a one-dimensional delay differential equation by using the Euler discretization. We first transform this class of delay difference equations into a high-dimensional discrete dynamical system. Then we prove that the map of the system is chaotic in the sense of both Devaney and Li-Yorke under some conditions, by employing the snap-back repeller theory. Finally, we give some computer simulations to illustrate the theoretical result.
\end{abstract}

MSC: 34C28; 37D45; 74H65

Keywords: chaos; delay difference equation; snap-back repeller; chaos in the sense of Devaney; chaos in the sense of Li-Yorke

\section{Introduction}

Time delay arises in many realistic systems with feedback in science and engineering. It is well known that time delays in feedback systems can have a considerable influence on the qualitative behavior of these systems. The existence of time delay may lead to some complex dynamic behaviors such as oscillation, divergence, bifurcation and chaos; see [1-4], and many references therein.

Recently, some researchers used the Euler discretization to explore the complex dynamical behaviors of nonlinear differential systems, such as determining the bifurcation diagrams with Hopf bifurcation, observing stable or unstable orbits, and chaotic behavior; see [3-6] etc. However, some complicated behaviors such as chaos which they observed were obtained only by numerical simulations, and have not been proved rigorously. It is noted that there could exist some false phenomena only by virtue of numerical simulations. Therefore, the existence of chaotic behavior of these systems needs to be studied rigorously.

In this paper, we study the chaotic behavior of the following delay difference equation:

$$
x(n+1)=\alpha x(n)+\beta x(n-k)\left(1-x^{2}(n-k)\right),
$$

where $\alpha, \beta$ are nonzero real parameters, and $k$ is a positive integer.

Equation (1.1) can be viewed as a discrete analogue of the following one-dimensional delay differential equation by using the forward Euler scheme

$$
\dot{x}(t)=-\mu x(t)+\gamma x(t-\tau)\left(1-x^{2}(t-\tau)\right),
$$

๑) 2013 Li et al.; licensee Springer. This is an Open Access article distributed under the terms of the Creative Commons Attribution License (http://creativecommons.org/licenses/by/2.0), which permits unrestricted use, distribution, and reproduction in any medium, provided the original work is properly cited. 
where $\mu>0$ and $\gamma$ are real parameters, $\tau>0$ is the delay. Equation (1.2) has been studied by $[7,8]$ for the periodic solutions, and it is a special case of the following equation:

$$
\dot{x}(t)=-\mu x(t)+f(x(t-\tau)),
$$

where $\mu>0, \tau>0$ is the delay, and $f$ is a one-dimensional nonlinear function. Equation (1.3) is known as the Mackey-Glass equation and has found many applications in physics [9], population dynamics [10], physiology [11], medicine [12], neural control [13], and economy [14].

The chaotic behaviors of equation (1.3) for some special forms of $f$ have been studied by many researchers. For example, Heiden and Mackey [15], and Walther [16], proved the existence of chaos in case of either piecewise constant or smooth but 'nearly' piecewise constant $f$. Lani-Wayda and Walther $[17,18]$ succeeded to prove chaos for certain smooth, nowhere constant $f$ (and assuming $\mu=0$ in (1.3)).

To the best of our knowledge, the research works on the chaotic behavior of equation (1.2) or its discrete analogue (1.1) with rigorously mathematical proof up to now are still few. The main purpose of this paper is to study the existence of chaos of equation (1.1) by using the snap-back repeller theory.

The rest of the paper is organized as follows. In Section 2, some basic concepts and lemmas are introduced. The transformation of the chaos problem is given in Section 3. In Section 4 , it is rigorously proved that there exists chaotic behavior in the delay difference equation by using the snap-back repeller theory. Finally, an illustrative example is provided with computer simulations.

\section{Preliminaries}

In this section, some basic concepts and lemmas are introduced.

In 1975, Li and Yorke [19] considered a one-dimensional continuous map and obtained the well-known result, that is, 'period 3 implies chaos. They first used the precise word 'chaos.' However, to the best of our knowledge, there is no unified definition of chaos in mathematics up to now. Depending on the requirements in different problems, there appeared several different definitions of chaos, some are stronger and some are weaker. For convenience, we present two definitions of chaos in the sense of Li-Yorke and Devaney.

Definition 2.1 Let $(X, d)$ be a metric space, $f: X \rightarrow X$ be a map, and $S$ be a set of $X$ with at least two distinct points. Then $S$ is called a scrambled set of $f$ if for any two distinct points $x, y \in S$,

(i) $\liminf _{n \rightarrow \infty} d\left(f^{n}(x), f^{n}(y)\right)=0$;

(ii) $\limsup _{n \rightarrow \infty} d\left(f^{n}(x), f^{n}(y)\right)>0$.

The map $f$ is said to be chaotic in the sense of Li-Yorke if there exists an uncountable scrambled set $S$ of $f$.

There are three conditions in the original characterization of chaos in Li-Yorke's theorem [19]. Besides the above conditions (i) and (ii), the third one is that for all $x \in S$ and for all periodic points $p$ of $f$,

$$
\limsup _{n \rightarrow \infty} d\left(f^{n}(x), f^{n}(p)\right)>0
$$


But conditions (i) and (ii) together imply that the scrambled set $S$ contains at most one point $x$ that does not satisfy the above condition. So, the third condition is not essential and can be removed.

Definition 2.2 [20] Let $(X, d)$ be a metric space and $V$ be a set of $X$. A map $f: V \rightarrow V$ is said to be chaotic on $V$ in the sense of Devaney if

(i) the set of the periodic points of $f$ is dense in $V$;

(ii) $f$ is topologically transitive in $V$;

(iii) $f$ has sensitive dependence on initial conditions in $V$.

In 1992, Banks et al. [21] proved that conditions (i) and (ii) together imply condition (iii) if $f$ is continuous in $V$ and $V$ is infinite. So, condition (iii) is redundant in the above definition. It has been proved that under some conditions, chaos in the sense of Devaney is stronger than that in the sense of Li-Yorke [22].

Remark 2.1 Some researchers consider that condition (i) in Definition 2.2 is not essential in the chaotic behavior. In 1990, Wiggins [23, Definition 4.11.2] called the map $f$ chaotic on $V$ if it satisfies conditions (ii) and (iii) in Definition 2.2. So, some researchers use the term 'chaos in the sense of Wiggins'. It is evident that chaos in the sense of Devaney is stronger than that in the sense of Wiggins.

In the following, by $B_{r}(z)$ and $\bar{B}_{r}(z)$ denote the open and closed balls of radius $r$ centered at $z \in X$, respectively. For convenience, we present some definitions in [24].

Definition 2.3 [24, Definitions 2.1-2.4] Let $(X, d)$ be a metric space and $f: X \rightarrow X$ be a map.

(i) A point $z \in X$ is called an expanding fixed point (or a repeller) of $f$ in $\bar{B}_{r}(z)$ for some constant $r>0$, if $f(z)=z$ and there exists a constant $\lambda>1$ such that

$$
d(f(x), f(y)) \geq \lambda d(x, y) \quad \forall x, y \in \bar{B}_{r}(z) .
$$

The constant $\lambda$ is called an expanding coefficient of $f$ in $\bar{B}_{r}(z)$. Furthermore, $z$ is called a regular expanding fixed point of $f$ in $\bar{B}_{r}(z)$ if $z$ is an interior point of $f\left(B_{r}(z)\right.$ ). Otherwise, $z$ is called a singular expanding fixed point of $f$ in $\bar{B}_{r}(z)$.

(ii) Assume that $z$ is an expanding fixed point of $f$ in $\bar{B}_{r}(z)$ for some $r>0$. Then $z$ is said to be a snap-back repeller of $f$ if there exists a point $x_{0} \in B_{r}(z)$ with $x_{0} \neq z$ and $f^{m}\left(x_{0}\right)=z$ for some positive integer $m$. Furthermore, $z$ is said to be a nondegenerate snap-back repeller of $f$ if there exist positive constants $\mu$ and $r_{0}<r$ such that $B_{r_{0}}\left(x_{0}\right) \subset B_{r}(z)$ and

$$
d\left(f^{m}(x), f^{m}(y)\right) \geq \mu d(x, y) \quad \forall x, y \in B_{r_{0}}\left(x_{0}\right) .
$$

$z$ is called a regular snap-back repeller of $f$ if $f\left(B_{r}(z)\right)$ is open and there exists a positive constant $\delta_{0}$ such that $B_{\delta_{0}}\left(x_{0}\right) \subset B_{r}(z)$ and for each positive constant $\delta \leq \delta_{0}$, $z$ is an interior point of $f^{m}\left(B_{\delta}\left(x_{0}\right)\right)$. Otherwise, $z$ is called a singular snap-back repeller of $f$. 
Remark 2.2 In 1978, Marotto [25] introduced the concept of snap-back repeller for maps in the Euclidean space $\mathbf{R}^{n}$. It is obvious that Definition 2.3 extends the concept of snapback repeller to maps in metric spaces. According to the above classifications of snap-back repellers for maps in metric spaces, the snap-back repeller in the Marotto paper [25] is regular and nondegenerate.

We now present two lemmas which will be used in Section 4. The following Lemma 2.1 is similar to that Lemma 3.5 in [26].

Lemma 2.1 Let $h:[-r, r] \subset \mathbf{R} \rightarrow \mathbf{R}$ be a continuously differentiable map. Assume that $h(0)=0, h^{\prime}(0) \neq 0$, then for a sufficiently small neighborhood $N$ of $0 \in \mathbf{R}$ and any bounded interval I of $\mathbf{R}$, there exists a positive constant $\beta^{*}:=\beta^{*}(N, I)$ such that the equation $\beta h(x)=$ $y$ has a solution $x \in N$ for any $|\beta|>\beta^{*}$ and $y \in I$.

Proof Since $h$ is continuously differentiable on $[-r, r]$, and $h(0)=0, h^{\prime}(0) \neq 0$, for any sufficiently small neighborhood $N \subset[-r, r]$ of 0 , there exist two neighborhoods $U$ and $V$ of 0 such that $U, V \subset N$ and $h: U \rightarrow V$ is a homeomorphism. In addition, for any bounded interval $I \subset \mathbf{R}$, there exists a positive constant $\beta_{0}$ such that

$$
\frac{1}{\beta_{0}} I:=\left\{\frac{1}{\beta_{0}} y: y \in I\right\} \subset V .
$$

Take $\beta^{*}:=\beta^{*}(N, I)=\beta_{0}$, then $\frac{1}{\beta} I \subset V$ for any $|\beta|>\beta^{*}$. Hence, for any $y \in I$ and $|\beta|>\beta^{*}$, the equation $h(x)=\frac{1}{\beta} y$, i.e., $\beta h(x)=y$ has a solution $x \in U \subset N$. This completes the proof.

Lemma 2.2 [27, Theorem 4.4] Letf $: \mathbf{R}^{n} \rightarrow \mathbf{R}^{n}$ be a map with a fixed point $z \in \mathbf{R}^{n}$. Assume that

(1) $f$ is continuously differentiable in a neighborhood of $z$ and all the eigenvalues of $D f(z)$ have absolute values larger than 1 , which implies that there exist a positive constant $r$ and a norm $\|\cdot\|$ in $\mathbf{R}^{n}$ such that $f$ is expanding in $\bar{B}_{r}(z)$ in $\|\cdot\|$;

(2) $z$ is a snap-back repeller of $f$ with $f^{m}\left(x_{0}\right)=z, x_{0} \neq z$, for some $x_{0} \in B_{r}(z)$ and some positive integer $m$. Furthermore, $f$ is continuously differentiable in some neighborhoods of $x_{0}, x_{1}, \ldots, x_{m-1}$, respectively, and $\operatorname{det} D f\left(x_{j}\right) \neq 0$ for $0 \leq j \leq m-1$, where $x_{j}=f\left(x_{j-1}\right)$ for $1 \leq j \leq m-1$.

Then for each neighborhood $U$ of $z$, there exist a positive integer $k>m$ and a Cantor set $\Lambda \subset$ $U$ such that $f^{k}: \Lambda \rightarrow \Lambda$ is topologically conjugate to the symbolic system $\sigma: \sum_{2}^{+} \rightarrow \sum_{2}^{+}$. Consequently, $f^{k}$ is chaotic on $\Lambda$ in the sense of Devaney and $f$ is chaotic in the sense of Li-Yorke. Further, there exists an uncountable, perfect and compact set $V \subset \mathbf{R}^{n}$, containing the Cantor set $\Lambda$, such that $f(V)=V$ and $f$ is chaotic on $V$ in the sense of Devaney.

Remark 2.3 In Lemma 2.2, it is only required that $f$ is continuously differentiable near $z$ and $x_{j}(0 \leq j \leq m-1)$. So, it is easy to be satisfied by many maps in practical models. The conclusions of Lemma 2.2 are slightly different from the original Theorem 4.4 in [27]. From [27, Theorem 4.4], we get that $f^{k}$ is chaotic on $\Lambda$ in the sense of Devaney. By [28, Lemma 2.4], we obtain that $f^{k}$ is chaotic in the sense of Li-Yorke. Consequently, $f$ is chaotic in the sense of Li-Yorke. The construction of $V$ and $f$ is chaotic on $V$ in the sense of Devaney can be conferred to [29, Theorem 4.2]. Under the conditions of Lemma 2.2, $z$ is a 
regular and nondegenerate snap-back repeller. Therefore, Lemma 2.2 can be briefly stated as the following: 'a regular and nondegenerate snap-back repeller in $\mathbf{R}^{n}$ implies chaos in the sense of both Devaney and Li-Yorke.' We refer to $[24,30]$ for details.

\section{Transformation of the chaos problem}

In this section, we will transform the delay difference equation (1.1) into a $(k+1)$-dimensional discrete dynamical system, and give some definitions about chaos of the two systems.

Let $u_{j}(n):=x(n+j-k-1)$ for $1 \leq j \leq k+1$, then system (1.1) can be transformed into the following $(k+1)$-dimensional discrete system

$$
u(n+1)=\left(\begin{array}{c}
u_{2}(n) \\
u_{3}(n) \\
\vdots \\
u_{k+1}(n) \\
\alpha u_{k+1}(n)+\beta u_{1}(n)\left(1-u_{1}^{2}(n)\right)
\end{array}\right):=F(u(n))
$$

where $u=\left(u_{1}, u_{2}, \ldots, u_{k+1}\right)^{T} \in \mathbf{R}^{k+1}$.

The map $F$ is said to be induced by $f$, and system (3.1) is said to be induced by system (1.1). It is evident that a solution $\{x(n-k), \ldots, x(n)\}_{n=1}^{\infty}$ of system (1.1) with an initial condition $\{x(-k), \ldots, x(0)\}$ corresponds to a solution $\{u(n)\}_{n=1}^{\infty}$ of system (3.1) with an initial condition $u(0)=\left(u_{1}(0), \ldots, u_{k+1}(0)\right)^{T} \in \mathbf{R}^{k+1}$. We call the solution $\{u(n)\}_{n=1}^{\infty}$ of (3.1) is induced by the solution $\{x(n-k), \ldots, x(n)\}_{n=1}^{\infty}$ of (1.1). Therefore, the dynamical behavior of system (1.1) is the same as that of its induced system (3.1) in $\mathbf{R}^{k+1}$. So, we introduce some relative concepts for system (1.1), which are motivated from some works in [30, Definitions 5.1 and 5.2].

\section{Definition 3.1}

(i) A point $z \in \mathbf{R}^{k+1}$ is called an $m$-periodic point of system (1.1) if $z \in \mathbf{R}^{k+1}$ is an $m$-periodic point of its induced system (3.1), that is, $F^{m}(z)=z$ and $F^{j}(z) \neq z$ for $1 \leq j \leq m-1$. In the special case of $m=1, z$ is called a fixed point or steady state of system (1.1).

(ii) A point $z \in \mathbf{R}^{k+1}$ is called a snap-back repeller of system (1.1) if $z \in \mathbf{R}^{k+1}$ is a snap-back repeller of its induced system (3.1).

(iii) The concepts of density of periodic points, topological transitivity, sensitive dependence on initial conditions, and the invariant set for system (1.1) are defined similarly to those for its induced system (3.1) in $\mathbf{R}^{k+1}$.

Definition 3.2 System (1.1) is said to be chaotic in the sense of Devaney (or Li-Yorke) on $V \subset \mathbf{R}^{k+1}$ if its induced system (3.1) is chaotic in the sense of Devaney (or Li-Yorke) on $V \subset \mathbf{R}^{k+1}$.

\section{Chaos in the system}

In this section, we will investigate the chaotic behavior of system (3.1), i.e., system (1.1), by showing that there exists a regular and nondegenerate snap-back repeller under some conditions. 
Consider the fixed points of system (3.1). From $F(\bar{u})=\bar{u}$, we obtain that system (3.1) has the following three fixed points for $\frac{1-\alpha}{\beta}<1$ :

$$
O:=(0, \ldots, 0)^{T}, \quad P:=(p, \ldots, p)^{T}, \quad Q:=(q, \ldots, q)^{T} \in \mathbf{R}^{k+1},
$$

where $p:=-\sqrt{1+\frac{\alpha-1}{\beta}}<0, q:=\sqrt{1+\frac{\alpha-1}{\beta}}>0$. We also get that $O$ is the unique fixed point of system (3.1) when $\frac{1-\alpha}{\beta} \geq 1$. In the two cases, $O$ is always a fixed point of system (3.1). So, in the following, we only show the fixed point $O$ can be a regular and nondegenerate snap-back repeller of system (3.1) under some conditions.

Theorem 4.1 There exists a positive constant $\beta_{0}$ such that for any $|\beta|>\beta_{0}$, the fixed point $O$ is a regular and nondegenerate snap-back repeller of system (3.1). Then system (3.1) and consequently, system (1.1), is chaotic in the sense of both Devaney and Li-Yorke.

Proof The idea in the proof is motivated by the proof of [26, Theorem 3.2]. We will apply Lemma 2.2 to prove this theorem. So, it suffices to show that all the assumptions in Lemma 2.2 are satisfied. It is clear that the map $F$ in system (3.1) is continuously differentiable in $\mathbf{R}^{k+1}$. So, $F$ satisfies the differentiability in Lemma 2.2 .

First, we show that there exists a positive constant $\beta_{1}$ such that $O$ is an expanding fixed point of the map $F$ in some norm in $\mathbf{R}^{k+1}$ for any $|\beta|>\beta_{1}$. In fact, the Jacobian matrix of $F$ at $O$ is

$$
D F(O)=\left(\begin{array}{ccccc}
0 & 1 & 0 & \cdots & 0 \\
0 & 0 & 1 & \cdots & 0 \\
\cdot & \cdot & \cdot & \cdots & \cdot \\
0 & 0 & 0 & \cdots & 1 \\
\beta & 0 & 0 & \cdots & \alpha
\end{array}\right)_{(k+1) \times(k+1)} .
$$

Its eigenvalues are determined by

$$
\lambda^{k+1}-\alpha \lambda^{k}-\beta=0 .
$$

Let $\beta_{1}:=1+|\alpha|$. From (4.1), we get that all the eigenvalues of $D F(O)$ have absolute values larger than 1 for any $|\beta|>\beta_{1}$. Otherwise, suppose that there exists an eigenvalue $\lambda_{0}$ of $D F(O)$ with $\left|\lambda_{0}\right| \leq 1$, then we get the following inequality:

$$
1+|\alpha| \geq\left|\lambda_{0}^{k+1}\right|+\left|\alpha \lambda_{0}^{k}\right| \geq\left|\lambda_{0}^{k+1}-\alpha \lambda_{0}^{k}\right|=|\beta|>1+|\alpha|,
$$

which is a contradiction. Hence, it follows from the first condition of Lemma 2.2 that there exists a positive constant $r$ and a norm $\|\cdot\|^{*}$ in $\mathbf{R}^{k+1}$ such that $O$ is an expanding fixed point of $F$ in $\bar{B}_{r}(O)$ in the norm $\|\cdot\|^{*}$, that is,

$$
\|F(x)-F(y)\|^{*} \geq \gamma\|x-y\|^{*} \quad \forall x, y \in \bar{B}_{r}(O),
$$

where $\gamma>1$ is an expanding coefficient of $F$ in $\bar{B}_{r}(O)$, and $\bar{B}_{r}(O)$ is the closed ball centered at $O \in \mathbf{R}^{k+1}$ of radius $r$ with respect to the norm $\|\cdot\|^{*}$. 
Next, we show that $O$ is a snap-back repeller of $F$ in the norm $\|\cdot\|^{*}$. Suppose that $W \subset$ $\bar{B}_{r}(O)$ is an arbitrary neighborhood of $O$ in $\mathbf{R}^{k+1}$, then there exists a small interval $U \subset \mathbf{R}$ containing 0 such that $\underbrace{U \times U \times \cdots \times U}_{k+1} \subset W$. In the following, we will show that there exists a positive constant $\beta_{2}$ such that for any $|\beta|>\beta_{2}$, there exists a point $O_{0} \in W$ with $O_{0} \neq O$ satisfying

$$
F^{k+2}\left(O_{0}\right)=O
$$

which implies that $O$ is a snap-back repeller of $F$.

For convenience, let $h(x):=x\left(1-x^{2}\right)$. It is clear that $h(x)$ is continuously differentiable on $\mathbf{R}$ and satisfies

$$
h(0)=0, \quad h^{\prime}(0)=1>0 .
$$

For $k=1$. From Lemma 2.1, it follows that there exists a positive constant $\beta_{2}^{\prime}$ such that for any $|\beta|>\beta_{2}^{\prime}$, there exist two points $x_{1}, x_{2} \in U$ satisfying the following equations:

$$
\left\{\begin{array}{l}
\beta h\left(x_{2}\right)=-\alpha \\
\beta h\left(x_{1}\right)=1-\alpha x_{2} .
\end{array}\right.
$$

Set $O_{0}=\left(x_{1}, x_{2}\right)^{T} \in \mathbf{R}^{2}$, then we get that $O_{0} \in U \times U \subset W$ with $O_{0} \neq O$ for any $|\beta|>\beta_{2}^{\prime}$. It follows from (4.2) that $F\left(O_{0}\right)=\left(x_{2}, 1\right)^{T}, F^{2}\left(O_{0}\right)=(1,0)^{T}, F^{3}\left(O_{0}\right)=O$.

For $k>1$, it also follows from Lemma 2.1 that there exists a positive constant $\beta_{2}^{*}$ such that for any $|\beta|>\beta_{2}^{*}$, there exist two points $x_{1}, x_{2} \in U$ satisfying the following equations:

$$
\left\{\begin{array}{l}
\beta h\left(x_{1}\right)=1 \\
\beta h\left(x_{2}\right)=-\alpha
\end{array}\right.
$$

Set $O_{0}=\left(x_{1}, x_{2}, 0, \ldots, 0\right)^{T} \in \mathbf{R}^{k+1}$, then we get that $O_{0} \in \underbrace{U \times U \times \cdots \times U}_{k+1} \subset W$ with $O_{0} \neq O$ for any $|\beta|>\beta_{2}^{*}$. It follows from (4.3) that $F\left(O_{0}\right)=\left(x_{2}, 0, \ldots, 1\right)^{T}, F^{j}\left(O_{0}\right)=$ $(0, \ldots, 0, \underbrace{1,0, \ldots, 0}_{j})^{T}$ for $2 \leq j \leq k+1$, and $F^{k+2}\left(O_{0}\right)=O$.

Take $\beta_{2}:=\max \left\{\beta_{2}^{\prime}, \beta_{2}^{*}\right\}$. Then for any $|\beta|>\beta_{2}$, there exists a point $O_{0} \in W$ with $O_{0} \neq O$ satisfying $F^{k+2}\left(O_{0}\right)=O$, in the two cases. Fix $\beta_{0} \geq \max \left\{\beta_{1}, \beta_{2}\right\}$. Then we get that $O$ is a snap-back repeller of $F$ for $|\beta|>\beta_{0}$.

Now, we shall show that for $|\beta|>\beta_{0}$,

$$
\operatorname{det} D F\left(O_{j}\right) \neq 0, \quad 0 \leq j \leq k+1 \text {, }
$$

where $O_{j}:=F\left(O_{j-1}\right)$ for $1 \leq j \leq k+1$. It is obvious that $h(x)$ is continuously differentiable on $\mathbf{R}$ and satisfies

$$
\begin{aligned}
& h(0)=h(1)=0, \\
& h^{\prime}(0)=1, \quad h^{\prime}(1)=-2 .
\end{aligned}
$$


Hence, from the second conclusion of (4.4), it follows that there exists a sufficiently small neighborhood $U_{1} \subset \mathbf{R}$ containing 0 such that $h^{\prime}(x) \neq 0$ for all $x \in U_{1}$. We can take sufficiently large $\beta_{0}$ such that $x_{1}, x_{2}$ obtained above also lie in $U_{1}$ for $|\beta|>\beta_{0}$ and satisfy equations (4.2) or (4.3). Consequently, we have

$$
h^{\prime}\left(x_{1}\right) \neq 0, \quad h^{\prime}\left(x_{2}\right) \neq 0 .
$$

A direct calculation shows that for any $u=\left(u_{1}, \ldots, u_{k+1}\right)^{T} \in \mathbf{R}^{k+1}$,

$$
\operatorname{det} D F(u)=(-1)^{k} \beta h^{\prime}\left(u_{1}\right) .
$$

For $k=1$, we get that $O_{0}=\left(x_{1}, x_{2}\right)^{T}, O_{1}=\left(x_{2}, 1\right)^{T}, O_{2}=(1,0)^{T} \in \mathbf{R}^{2}$. It follows from the second conclusion of (4.4), (4.5) and (4.6) that for $|\beta|>\beta_{0}$,

$$
\operatorname{det} D F\left(O_{j}\right) \neq 0, \quad \text { for } 0 \leq j \leq 2 \text {. }
$$

For $k>1$, we get that $O_{0}=\left(x_{1}, x_{2}, 0, \ldots, 0\right)^{T}, O_{1}=\left(x_{2}, 0, \ldots, 0,1\right)^{T}$, and $O_{j}=(0, \ldots, 0$, $\underbrace{1,0, \ldots, 0}_{j})^{T} \in \mathbf{R}^{k+1}$ for $2 \leq j \leq k+1$. Hence, from the second conclusion of (4.4), (4.5) and (4.6), we get that for $|\beta|>\left|\beta_{0}\right|$,

$$
\operatorname{det} D F\left(O_{j}\right) \neq 0, \quad \text { for } 0 \leq j \leq k+1 \text {. }
$$

Therefore, all the assumptions in Lemma 2.2 are satisfied and $O$ is a regular and nondegenerate snap-back repeller of system (3.1). Therefore, system (3.1), i.e., system (1.1), is chaotic in the sense of both Devaney and Li-Yorke. The proof is complete.

Remark 4.1 From the proof of Theorem 4.1, we see there exists some positive constant $\beta_{0}$ such that for any $|\beta|>\beta_{0}$, system (3.1) is chaotic in the sense of both Devaney and LiYorke. However, it is very difficult to determine the concrete value $\beta_{0}$ since the expanding area of a fixed point is not easy to obtain. This will be left for our further research.

Remark 4.2 The system (3.1) consists of a $k$-dimensional linear subsystem and onedimensional nonlinear subsystem. That is, the folding and stretching only occurs in the variable $u_{k+1}$, and all the other variables $u_{j}$ are replaced by $u_{j+1}$ for $1 \leq j \leq k$. So, system (3.1) can be viewed as one of the simplest systems that can show higher-dimensional chaos. Consequently, system (1.1) can be viewed as one of the simplest delay difference systems that show chaos.

In order to help better visualize the theoretical result, two computer simulations are done, which exhibit complicated dynamical behaviors of the induced system (3.1), i.e., system (1.1). We take $\alpha=-0.1, \beta=2.7, k=1,2$ for computer simulations. It follows from the proof of Theorem 4.1 that $O$ is an expanding fixed point of system (3.1) when $|\beta|>$ $1+|\alpha|=1$.1. Furthermore, there exists some positive constant $\beta_{0}>1.1$ such that for $|\beta|>$ $\beta_{0}$, we have two couples of points $x_{1} \approx 0.4879, x_{2} \approx 0.0371$ satisfying equation (4.2) for $k=1$, and $x_{1} \approx 0.4832, x_{2} \approx 0.0371$ satisfying equation (4.3) for $k>1$. Consequently, $O$ is a regular and nondegenerate snap-back repeller of system (3.1) for $|\beta|>\beta_{0}$. Therefore, 


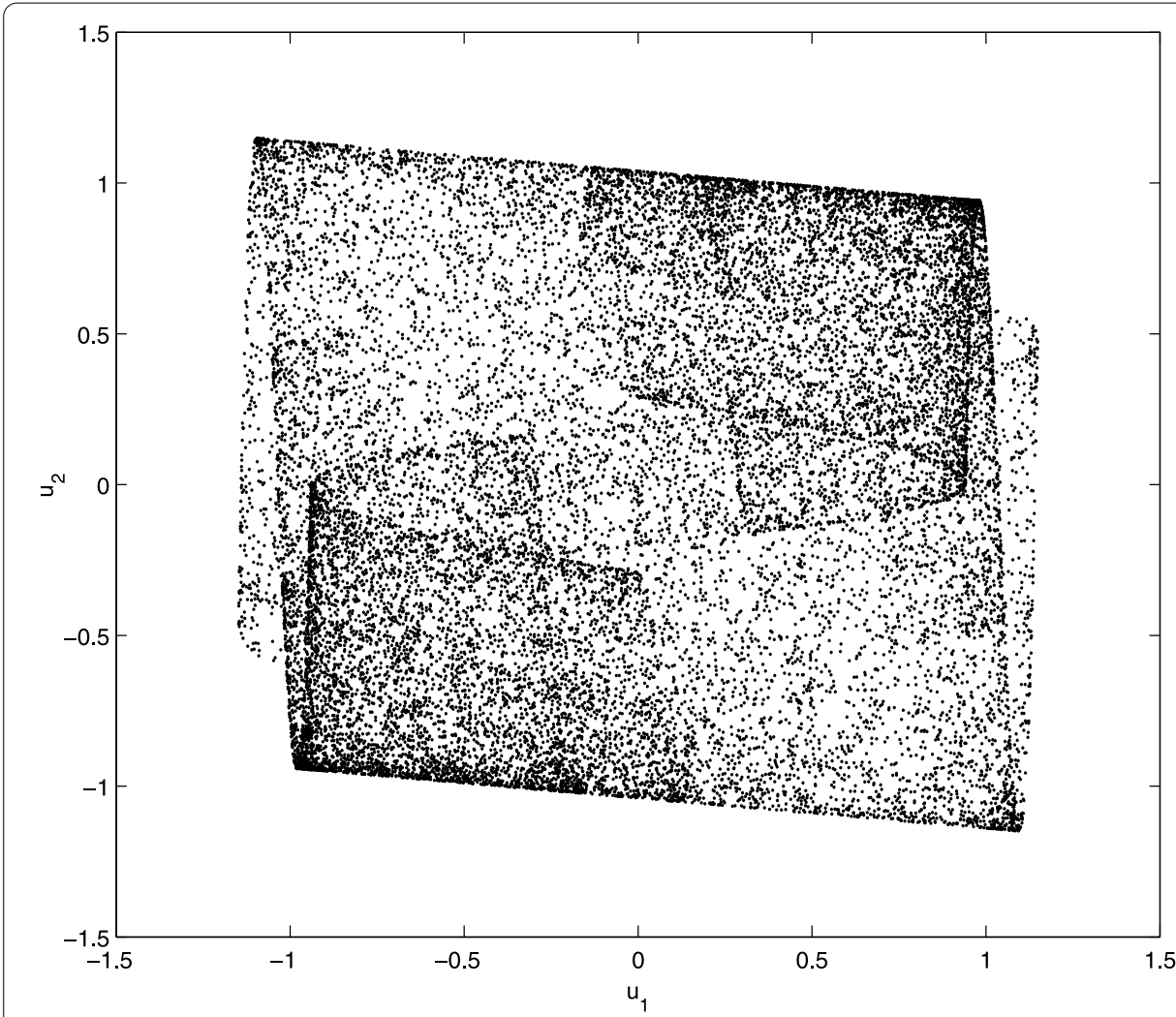

Figure 1 A dense orbit of system (3.1) in the $\left(u_{1}, u_{2}\right)$ space for $\alpha=-0.1, \beta=2.7, k=1$, and $n$ from 0 to 20,000 , with the initial condition $u(0)=(0.01,0.01)^{T}$.



Figure 2 A dense orbit of system (3.1) in the $\left(u_{1}, u_{2}, u_{3}\right)$ space for $\alpha=-0.1, \beta=2.7, k=2$, and $n$ from 0 to 20,000 , with the initial condition $u(0)=(0.01,0.01,0.01)^{T}$. 
system (3.1), i.e., system (1.1), is chaotic in the sense of both Devaney and Li-Yorke. Some simulation results are shown in Figures 1-2 for $k=1,2$ and $n$ from 0 to 20,000, respectively. It is a dense orbit in each picture, which clearly shows that the system has very complicated dynamical behaviors.

\section{Competing interests}

The authors declare that they have no competing interests.

\section{Authors' contributions}

ZL carried out the main part of this manuscript. QZ and DL participated in discussion. All authors read and approved the final manuscript.

\section{Acknowledgements}

This work was supported by the National Natural Science Foundation of China (Grant Nos. 11101246 and 11101247).

Received: 4 December 2012 Accepted: 23 March 2013 Published: 10 April 2013

\section{References}

1. Busenbrg, S, Martelli, M: Delay Differential Equations and Dynamical Systems. Springer, Berlin (1991)

2. Jiang, M, Shen, Y, Jian, J, Liao, X: Stability, bifurcation and a new chaos in the logistic differential equation with delay. Phys. Lett. A 350, 221-227 (2006)

3. Peng, MS, Uçar, A: The use of the Euler method in identification of multiple bifurcations and chaotic behavior in numerical approximations of delay differential equations. Chaos Solitons Fractals 21, 883-891 (2004)

4. Peng, MS, Yuan, Y: Stability, symmetry-breaking bifurcation and chaos in discrete delayed models. Int. J. Bifurc. Chaos 18, 1477-1501 (2008)

5. He, ZM, Lai, X: Bifurcation and chaotic behavior of a discrete-time predator-prey system. Nonlinear Anal., Real World Appl. 12, 403-417 (2011)

6. Fan, DJ, Wei, JJ: Bifurcation analysis of discrete survival red blood cells model. Commun. Nonlinear Sci. Numer. Simul. 14, 3358-3368 (2009)

7. Kaplan, JL, Yorke, JA: Ordinary differential equations which yield periodic solutions of differential delay equations. J. Math. Anal. Appl. 48, 317-324 (1974)

8. Dormayer, $\mathrm{P}$ : The stability of special symmetric solutions of $\dot{x}=\alpha f(x(t-1))$ with small amplitudes. Nonlinear Anal., Theory Methods Appl. 14, 701-715 (1990)

9. Furomochi, T: Existence of periodic solutions of one-dimensional differential-delay equations. Tohoku Math. J. 30 13-35 (1978)

10. Gurney, WSC, Blythe, SP, Nisbeth, RM: Nicholson's blowflies revisited. Nature 287, 17-21 (1978)

11. Heiden, U: Delays in physiological systems. J. Math. Biol. 8, 345-364 (1979)

12. Mackey, MC, Glass, L: Oscillation and chaos in physiological control systems. Science 197, $287-289$ (1977)

13. Mackey, MC, Heiden, U: The dynamics of recurrent inhibition. J. Math. Biol. 19, 211-225 (1984)

14. Mackey, MC: Commodity price fluctuations: price dependent delays and nonlinearities as explanatory factors. J. Econ. Theory 48, 497 (1990)

15. Heiden, U, Mackey, MC: The dynamics of production and destruction: analytic insight into complex behavior. J. Math Biol. 16, 75-101 (1982)

16. Heiden, U, Walther, HO: Existence of chaos in control systems with delayed feedback. J. Differ. Equ. 47, $273-295$ (1983)

17. Lani-Wayda, B, Walther, HO: Chaotic motion generated by delayed negative feedback, Part I: a transversality criterion. Differ. Integral Equ. 8, 1407-1452 (1995)

18. Lani-Wayda, B, Walther, HO: Chaotic motion generated by delayed negative feedback, Part II: construction of nonlinearities. Math. Nachr. 180, 141-211 (1996)

19. Li, TY, Yorke, JA: Period three implies chaos. Am. Math. Mon. 82, 985-992 (1975)

20. Devaney, RL: An Introduction to Chaotic Dynamical Systems. Addison-Wesley, New York (1987)

21. Banks, J, Brooks, J, Cairns, G, Davis, G, Stacey, P: On Devaney's definition of chaos. Am. Math. Mon. 99, 332-334 (1992)

22. Huang, W, Ye, XD: Devaney's chaos or 2-scattering implies Li-Yorke's chaos. Topol. Appl. 117, 259-272 (2002)

23. Wiggins, S: Introduction to Applied Nonlinear Dynamical Systems and Chaos. Springer, New York (1990)

24. Shi, YM, Chen, GR: Chaos of discrete dynamical systems in complete metric spaces. Chaos Solitons Fractals 22 , 555-571 (2004)

25. Marotto, FR: Snap-back repellers imply chaos in $R^{n}$. J. Math. Anal. Appl. 63, 199-223 (1978)

26. Huang, Y, Zou, XF: Co-existence of chaos and stable periodic orbits in a simple discrete neural network. J. Nonlinear Sci. 15, 291-303 (2005)

27. Shi, YM, Chen, GR: Discrete chaos in Banach spaces. Sci. China Ser. A 34, 595-609 (2004); (English version 48, 222-238 (2005))

28. Shi, YM, Yu, P: Chaos induced by regular snap-back repellers. J. Math. Anal. Appl. 337, 1480-1494 (2008)

29. Shi, YM, Yu, P: Study on chaos by turbulent maps in noncompact sets. Chaos Solitons Fractals 28, 1165-1180 (2006)

30. Shi, YM, Yu, P, Chen, GR: Chaotification of dynamical systems in Banach spaces. Int. J. Bifurc. Chaos 16, 2615-2636 (2006)

doi:10.1186/1687-1847-2013-99

Cite this article as: Li et al.: Chaotic behavior in a class of delay difference equations. Advances in Difference Equations 2013 2013:99. 